\title{
О ТРУДНОСТЯХ ДИАГНОСТИКИ И ЛЕЧЕНИЯ РАКА ОКОЛОЩИТОВИДНОЙ ЖЕЛЕЗЫ
}

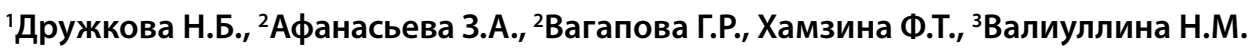

'ГАУЗ «Республиканский клинический онкологический диспансер» Министерства здравоохранения Республики Татарстан

2Казанская государственная медицинская академия — филиал ФГБОУ ДПО РМАНПО Минздрава России

ЗГАУЗ «Межрегиональный клинико-диагностический чентр» Министерства здравоохранения Республики Татарстан, заведующий лабораторией радиоизотопной диагностики

АКТУАЛЬНОСТЬ: рак околощитовидной железы (ОЩЖ) - редкий, высоко агрессивный тип эндокринного рака с частотой встречаемости менее 1 случая на 1 млн. населения. Среди пациентов с первичным гиперпаратиреозом (ПГПТ) карцинома ОЩЖ встречается в 0,5-5\% случаев. Прогноз для больных данной опухолью вариативен и зависит от распространенности процесса и радикальности выполненной операции. Рецидив рака ОЩЖ встречается более чем у половины больных в среднем через 3 года от первичной операции, что требует повторного хирургического вмешательства или медикаментозной терапии, направленной на коррекцию гиперкальциемии.

ЦЕЛЬ: оценить опыт диагностики и лечения рака ОЩЖ в Республике Татарстан в период с 2008 по 2019 гг.

МАТЕРИАЛЫ И МЕТОДЫ: ретроспективно изучены истории болезни и амбулаторные карты 8 пациентоВ, проходивших лечение в ГАУЗ РКОД МЗ РТ с 2008 по 2019 гг.

РЕЗУЛЬТАТЫ: среди пациентов было 2 мужчин в возрасте 35 и 50 лет и 6 женщин в возрасте 29-76 лет. У 6 пациентов был манифестный гиперпаратиреоз с костными и костно-висцеральными проявлениями, 2 были госпитализированы с диагнозом опухоли щитовидной железы и симптомами масс-эффекта. Уровень паратгормона (ПТГ) варьировал от 350 до 3227 пг/мл (N-15-65), уровень общего кальция (Са) — от 2,77 до 3,35 ммоль/л (N-2,0-2,57). По результатам топической диагностики (УЗИ, сцинтиграфия ОЩЖ, РКТ шеи и органов грудной полости) у 6 пациентов паратиромы располагались на шее, у 1 - загрудинно, у 1 в верхнем средостении. Все пациенты получили хирургическое лечение: 5 пациентам выполнена гемитиреоидэктомия с удалением опухоли ОЩЖ; 1 - тиреоидэктомия с удалением опухоли ОЩЖ и центральная лимфодиссекция; 1 - цервикотомия, стернотомия, удаление опухоли ОЩЖ; 1 - видеоторакоскопия, удаление опухоли ОЩЖ. В послеоперационном периоде у 3 пациентов уровень ПтГ и Са снизился до референсных значений нормы, у 5 - развился гипопаратиреоз, потребовавший назначения препаратов кальция и активных метаболитов витамина Д. Гистологически и иммуногистохимически у всех больных верифицирован рак ОЩЖ. На протяжении 6-11 лет живы 7 пациентов. Рецидивы рака ОЩЖ, манифестировавшие повышением уровня ПТГ, общего и ионизированного крови, возникли у 2 пациентов. У одного пациента с Кі-67=8\% через 5 лет после операции диагностированы метастазы в паратрахеальных лимфоузлах. У другой пациентки с Ki-67=12\% через 1,5 года после операции найдены множественные метастазы в костях и верхней доле правого легкого, впоследствии верифицированные гистологически. Пациенту выполнена селективная шейная лимфодиссекция (6 уровень), пациентке - атипическая резекция верхней доли правого легкого, ламинэктомия С5, остеофиксация тел С4-С6 позвонков. Сохраняющаяся тяжелая гиперкальциемия после оперативного лечения у пациентки была успешно корригирована цинакальцетом и деносумабом на протяжении 9 месяцев. Пациентка скончалась через 3 года после первичной операции вследствие острой почечной недостаточности, развившейся на фоне гиперкальциемического криза.

ВЫВОды: диагностика рака ОЩЖ и его рецидивов является трудной задачей для практикующего врача. Основным методом лечения остается хирургический. Гиперкальциемия, развивающаяся при диссеминации процесса, требует постоянной медикаментозной коррекции антирезорбтивными препаратами и кальцимиметиками.

КЛЮчЕВЫЕ СЛОВА: рак околощитовидной железы, гиперкальциемия, цинакальцет, деносумаб. 